\title{
Intervenção Educativa no Cuidado Obstétrico através de um Aplicativo para Dispositivos Móveis: APP Meu Pré-natal
}

\author{
Zilma S Nogueira Reis ${ }^{1}$ \\ Júlia Relva Basso ${ }^{1}$ \\ Marcelo R. Santos Junior ${ }^{1}$ \\ Isaias José Ramos de Oliveira ${ }^{1}$ \\ Wagner Bento Magalhães ${ }^{1}$ \\ Juliano de Souza Gaspar ${ }^{1}$ \\ Alamanda Kfoury Pereira ${ }^{1}$ \\ ${ }^{1}$ Universidade Federal de Minas Gerais, Brasil; E-Mails: zilma.medicina@gmail.com/zilma@medicina.ufmg.br; \\ isaias@medicina.ufmg.br; julianogaspar@gmail.com.
}

\begin{abstract}
Resumo
Introdução: A comunicação entre os profissionais de saúde e seus pacientes tem se beneficiado da popularização dos dispositivos móveis. Essa análise tem por objetivo apresentar um aplicativo direcionado para mulheres durante a gravidez, parto e puerpério e os resultados da sua disseminação na sociedade. Metodologia: Caracterizado como pesquisa extensionista, o projeto APP - Meu Pré-natal é uma ação acadêmica voltada para sociedade. O desenvolvimento do software foi conduzido por uma equipe multidisciplinar da área das ciências da saúde e da computação. O conteúdo elaborado e validado disponibilizou textos de base cientifica em português, inglês e espanhol com linguagem acessível ao público leigo, imagens e vídeo, através de interface para navegação fácil e intuitiva entre seus módulos. O software de desenvolvimento híbrido foi disponibilizado gratuitamente em lojas de aplicativos na Internet. Resultados: Entre outubro de 2016 a junho de 2017 o aplicativo foi baixado 19.364 vezes. A maior parte a partir de dispositivos de comunicação do tipo Android: 14.791 (76,4\%). Os usuários foram provenientes de 81 países. Conclusões: A rápida popularização e o alcance do aplicativo deixam claro a importância desse canal de comunicação para propostas de educação em saúde e a valorização da certificação acadêmica de seu conteúdo pelo usuário.
\end{abstract}

Palavras-chave: dispositivos móveis; educação em saúde; pré-natal; relações comunidade-instituição.

\begin{abstract}
Introduction: Communication between health professionals and their patients has benefited with the popularization of mobile devices. This analysis intends to present an application aimed to women during pregnancy, childbirth and the puerperium period and set out the results of their dissemination in society. Methodology: Characterized as extension research, the APP - Meu Pre-natal project is an academic action focused on community spreading. Software development was conducted by a multidisciplinary team in the area of health and computing sciences. The content developed and validated provided scientifically-based texts in Portuguese, English and Spanish with language accessible to the general public, images and video, through an interface for easy and intuitive navigation among its modules. Hybrid development software was employed, and it is available for free from Internet application stores. Results: Between October 2016 and June 2017 the application was downloaded 19,364 times. Most from Android-type communication devices: 14,791 (76.4\%). Users came from 81 countries. Conclusions: The
\end{abstract}


rapid popularization and application scope make clear the importance of this communication channel for proposals of health education and the valorization of the academic certification of its contents by the user.

Keywords: community-institution relations; health education; mobile devices; prenatal.

\section{Introdução}

A rápida disseminação dos aplicativos (APP) para dispositivos móveis em todo mundo tem impulsionado a democratização da informação, com perspectivas de se tornarem, em pouco tempo o principal guia de consulta sobre o cuidado em saúde (Grundy, Wang, \& Bero, 2016). Essa tecnologia traz de forma intrínseca o potencial de conectar pessoas, mas em especial atinge adolescentes e adultos jovens (Chen \& Mangone, 2016), um público de especial interesse para se fomentar o cuidado obstétrico.

Durante a gestação, período de grandes transformações biológicas, psíquicas e sociais entre os casais, é fundamental que a gestante e seu feto estejam posicionados no centro da prestação do cuidado (Patah \& Malik, 2011). Tal atitude demanda mudanças de postura nas relações entre a equipe de saúde e o casal, de forma a permitir sua participação ativa. Essa mudança de paradigma pode em muito se beneficiar de um amplo acesso à informação confiável sobre a gravidez por outros canais de comunicação, desde que validados e confiáveis. 0 processo assistencial em obstetrícia passa pela prevenção de agravos, promoção de hábitos saudáveis de vida, ultrapassando a presença em seis consultas tradicionais, preconizadas durante o cuidado pré-natal (Laurenti et al., 2013). Inúmeras práticas rotineiras carecem de orientações específicas na gravidez: alimentação saudável, cuidados relativos à saúde ocupacional, prática de esportes, exposição a agentes nocivos como medicamentos sintomáticos e outros potencialmente teratogênicos, exposição às arboviroses, para exemplificar um tema da atualidade (Brasil, Saúde, \& Estratégicas, 2007; Brasil et al., 2016). Além disto, os modelos de assistência obstétrica sofrem influências de seu tempo, sejam no aspecto da compreensão e conhecimento da fisiologia da mulher, sejam sob o ponto de vista sociocultural, regional, ético e econômico (Patah \& Malik 2011). Acredita-se que a informação oportuna e de qualidade possa reduzir conflitos sustentados por mitos e inverdades sobre a saúde, em especial sobre a gravidez e apoiar o cuidado (Vettore \& Lamarca, 2012).

Na vertente impulsionada pela Internet das Coisas, os dispositivos móveis vêm experimentando um crescimento expressivo (Islam et al., 2015), permitindo novos recursos computacionais e garantindo a presença de aplicativos (APP) em inúmeros cenários da atenção à saúde (Tibes, Dias, \& Zem-Mascarenhas, 2014). Tais abordagens, mesmo que ainda sem regulamentação em muitos países e sem validação de seus conteúdos, trazem uma nova maneira dos médicos e outros especialistas se comunicarem com a população (Grundy, Wang, \& Bero, 2016). Com facilidade, os APPs são programados para monitorar doenças crônicas, oferta recursos interativos, fazendo com que o próprio individuo assuma um papel central no autocuidado. Ao mesmo tempo, permite que a melhor orientação sobre saúde esteja cada vez mais presente em sua vida, próximo às suas escolhas cotidianas (Fonseca \& de Menezes Alencar, 2016).

O Centro de Informática em Saúde da Faculdade de Medicina da Universidade Federal de Minas Gerais (CINS) atua no processo interdisciplinar educativo para promover uma interação transformadora na sociedade, através da disseminação da informação em saúde, envolvendo a tecnologia da informação e comunicação (TIC). O projeto Meu Pré-natal teve origem em uma disciplina de graduação regular no curriculum médico desta Faculdade, a Obstetrícia Básica. Envolveu estudantes de medicina, professores deste curso e do curso de enfermagem, de forma integrada aos profissionais da computação do CINS e pós-graduandos do Programa Saúde da Mulher. A presente análise tem por objetivo apresentar o desenvolvimento de um APP educativo para uso em dispositivos móveis, direcionado para apoiar as mulheres durante a gravidez, parto e puerpério e os resultados da sua disseminação na sociedade.

\section{Metodologia}

O Projeto caracterizou-se como pesquisa extensionista, utilizando-se de um APP gratuito, a ser livremente instalado pela própria gestante interessada, para disseminar informações de qualidade sobre a gravidez. A proposta recebeu o nome de "Meu Pré-natal - Aplicativo para dispositivos móveis", sendo registrada no Sistema de Informação da 
Extensão da Universidade Federal de Minas Gerais (UFMG), sob o número 402465. Foi albergada como uma das linhas de ação do Programa Multidisciplinar de Estudos sobre o Parto Seguro: promovendo conhecimento, qualificação e diretrizes acadêmicas para a população. Esse programa reúne um conjunto de ações fundamentadas na vertente educativa e transformadora do acesso à informação sobre as melhores práticas assistenciais na gravidez.

Em relação ao desenvolvimento do APP, uma equipe multidisciplinar formada por professores, profissionais da computação e alunos da Faculdade de Medicina da UFMG participou das etapas de projeto, execução, divulgação e monitoramento do programa de computador. No que se refere ao seu propósito, o acesso direto às melhores práticas de assistência a gestação constituiu o direcionamento central na preparação dos conteúdos e do sistema.

De forma estratégica a principal causa de morte neonatal, o nascimento prematuro (Grünebaum et al., 2014) foi colocada como tema direcionador do cuidado preventivo. Uma vez que a idade gestacional é o principal indicador de sobrevida do recém-nascido (Young et al., 2017), um gestograma com o cálculo da cronologia da gravidez compõe a interface principal com os usuários. 0 cálculo da data provável do parto foi obtido somando-se 280 dias à partir da data da última menstruação informada pela gestante (Moraes \& Reichenheim 2000) e pelo ultrassom, considerando-se ainda que aquele realizado no primeiro trimestre gestacional é o referencial padrão-ouro para tal estimativa (Butt et al., 2014; ACOG, 2014). A partir destes dois marcos informados pelo usuário do APP, a idade gestacional apresentada em semanas completas e dias foi automatizada através da diferença simples entre a data do acesso e as datas da última menstruação e ultrassom. Para garantir a qualidade destas informações, a data informada é preferencialmente aquela calculada pelo ultrassom precoce, até 13 semanas. A margem de erro da estimativa foi disponibilizada, segundo consenso científico (Butt et al., 2014; ACOG, 2014).

Caracterizado na modalidade de APP educativo em saúde, o casal grávido e principalmente a gestante tem disponível, além das informações sobre a trajetória de sua gravidez desde o momento que se inicia até o pós-parto, várias funcionalidades como contador de contrações e vídeo sobre o parto. Para isso, temas relevantes sobre o modelo de assistência obstétrica, dados essenciais sobre a gestação, cuidados preventivos e sinais de alerta foram preparados por alunos de graduação da Disciplina de Obstetrícia Básica, sob supervisão docente. Na etapa de preparação do conteúdo, estudantes de medicina listaram as dúvidas mais frequentes das gestantes, apontadas durante as consultas no ambulatório de pré-natal, durante a disciplina de prática. Os temas abordados foram agrupados cronologicamente e por relevância para apontamentos de gravidade. Em seguida, orientados por professores do Departamento de Ginecologia e Obstetrícia e da Escola de Enfermagem da UFMG, respostas baseadas na melhor evidência científica e nas recomendações das políticas públicas brasileiras e internacionais foram alocadas para cada questão (UNICEF, 2011a; Brasil, 2014a; Souza, 2017; UNICEF, 2011b). O tamanho máximo de uma resposta foi ajustado para 140 caracteres, tomando como base o tamanho de uma mensagem no Twitter (Kwak et al., 2010), sem prejuízo ao conteúdo. Em seguida, o conjunto de perguntas e respostas foi validado e ajustado quanto à linguagem utilizada, entendimento pelo público-alvo e relevância dos temas, através de entrevista direta com gestantes atendidas no Pré-natal do Hospital das Clínicas da UFMG. Um grupo de oito estudantes de medicina analisou um conjunto de itens junto às gestantes voluntárias em sala de espera para consulta médica. A etapa final foi a revisão de todo conteúdo por especialistas acadêmicos sendo eles: quatro professores doutores, três deles em obstetrícia e um em enfermagem obstétrica. Parte dos itens foi incorporado em um roteiro para preparação de uma mídia em vídeo-animação.

Pensado como um sistema eletrônico de informação, o software foi preparado para atingir o maior número possível de gestantes, em todas as classes sociais e sistemas operacionais mais usados na atualidade. A Tabela 1 apresenta os principais requisitos funcionais e não funcionais do APP. O desenho do produto teve como foco a divulgação dos conteúdos científicos em linguagem acessível às gestantes, buscando garantir a melhor qualidade de aprendizagem ao público alvo.

Tabela 1. Requisitos funcionais e não funcionais do APP Meu Pré-natal.

\begin{tabular}{ll}
\hline Código & Requisitos funcionais \\
\hline RF01 & Agrupar as funcionalidades do sistema em módulos de fácil acesso. \\
\hline RF02 & Permitir cadastro de uma gestante com suas informações básicas. \\
\hline RF03 & $\begin{array}{l}\text { Calcular a Data Provável do Parto (DPP) a partir da Data da Última Menstruação (DUM) e informar } \\
\text { o erro esperado (Butt et al. 2014, Moraes and Reichenheim 2000). }\end{array}$ \\
\hline
\end{tabular}




\begin{tabular}{|c|c|}
\hline RF04 & $\begin{array}{l}\text { Calcular a Data Provável do Parto (DPP) a partir do primeiro exame de Ultrassom e informar o } \\
\text { erro esperado ACOG (2014). }\end{array}$ \\
\hline RF05 & Exibir a DPP e um calendário com os meses de gestação através de um gestograma. \\
\hline RF06 & Permitir que a gestante receba notificações acerca dos marcos mais importantes da gestação. \\
\hline RF07 & $\begin{array}{l}\text { Permitir a coleta de dados referentes ao parto: data, o peso do bebê, a nota de Apgar, o local onde } \\
\text { nasceu. }\end{array}$ \\
\hline RF08 & Permitir upload de fotos. \\
\hline RF09 & Permitir acesso a vídeos educativos. \\
\hline RF10 & $\begin{array}{l}\text { Permitir acesso às informações confiáveis e educativas sobre a gestação, o parto e o pós-parto, na } \\
\text { forma de perguntas e respostas. }\end{array}$ \\
\hline RF11 & Disponibilizar índice para busca por palavra para perguntas e respostas do sistema. \\
\hline RF12 & $\begin{array}{l}\text { Permitir compartilhamento do nascimento do bebê, contendo nome do bebê, a data de nascimento, } \\
\text { peso, local de nascimento e foto. }\end{array}$ \\
\hline RF13 & Informar as fontes bibliográficas para o conteúdo técnico-científico. \\
\hline RF14 & Permitir estatística remota de utilização do aplicativo. \\
\hline Código & Requisitos não funcionais \\
\hline RNF01 & Acessível apenas através de aplicativo instalado em dispositivo móvel, modo on line e off line. \\
\hline RNF02 & Ser de acesso livre e gratuito. \\
\hline RNF03 & Desenvolvimento com linguagens e softwares open-source. \\
\hline RNF05 & Ser de fácil preenchimento. \\
\hline RNF06 & Apresentar requisitos para ser oferecido em lojas virtuais de aplicativos. \\
\hline RNF07 & Ser compatível com sistemas Android e iOS. \\
\hline RNF08 & Ser modular, de forma que possibilite futura expansão. \\
\hline RNF09 & $\begin{array}{l}\text { Reuniões periódicas com a equipe de saúde para promover o engajamento e contribuições para o } \\
\text { desenvolvimento do aplicativo. }\end{array}$ \\
\hline RNF10 & Exibir a logomarca dos patrocinadores, o registro na Universidade e o registro de propriedade intelectual. \\
\hline
\end{tabular}

Quanto aos métodos computacionais, a plataforma open-source IONIC para desenvolvimento híbrido foi empregada. O framework foi desenvolvido sobre o AngularJS e Apache Cordova, de forma a ser beneficiado por ferramentas e serviços para um desenvolvimento multiplataforma por tecnologia web, como: Cascading Style Sheets, ou Folhas de Estilo em Cascata (CSS), HyperText Markup Language, ou Linguagem de Marcação de HiperTexto5 (HTML5) e Syntactically Awesome Stylesheets (SAS). Toda a programação realizada através dessas tecnologias ocorreu por meio de uma ponte realizada pelo JavaScript com o framework da aplicação, que por sua vez gerou o código para as respectivas arquiteturas e sistemas operacionais desejados. Desta forma o APP foi publicado nas lojas de aplicativos nativas do projeto: App Store (iOS), Google Play (Android OS) na categoria Saúde e Bem Estar (Grundy, Wang, \& Bero, 2016).

Quanto à divulgação da aplicação, acessibilidade na Internet e monitoramento pós-implantação, a fase de testes on line se iniciou em 15/07/2016. A publicação oficial ocorreu em 1/10/2016, sendo disponibilizado gratuitamente através das lojas de aplicativos Google Inc. e Apple Inc. Os resultados aqui apresentados se referem ao período até 30/04/2017. A base de dados encontra-se nos servidores do CINS da Faculdade de Medicina da UFMG, responsável também pela segurança, sigilo da informações, pelo monitoramento de sua disseminação, ajustes e versionamento a cada nova melhoria.

\section{Resultados}

Durante a primeira instalação, um Termo de Adesão apresenta a proposta o projeto, sua vinculação acadêmica e informa aos usuários o acesso aos autores registrados no Sistema de Extensão da UFMG, além dos créditos aos patrocinadores. A interface principal do APP traz um gestograma dinâmico e automatizado que apresenta a 
cronologia da gravidez a partir dos dois referenciais clínicos: a data da última menstruação e o primeiro ultrassom obstétrico (Figura 1).

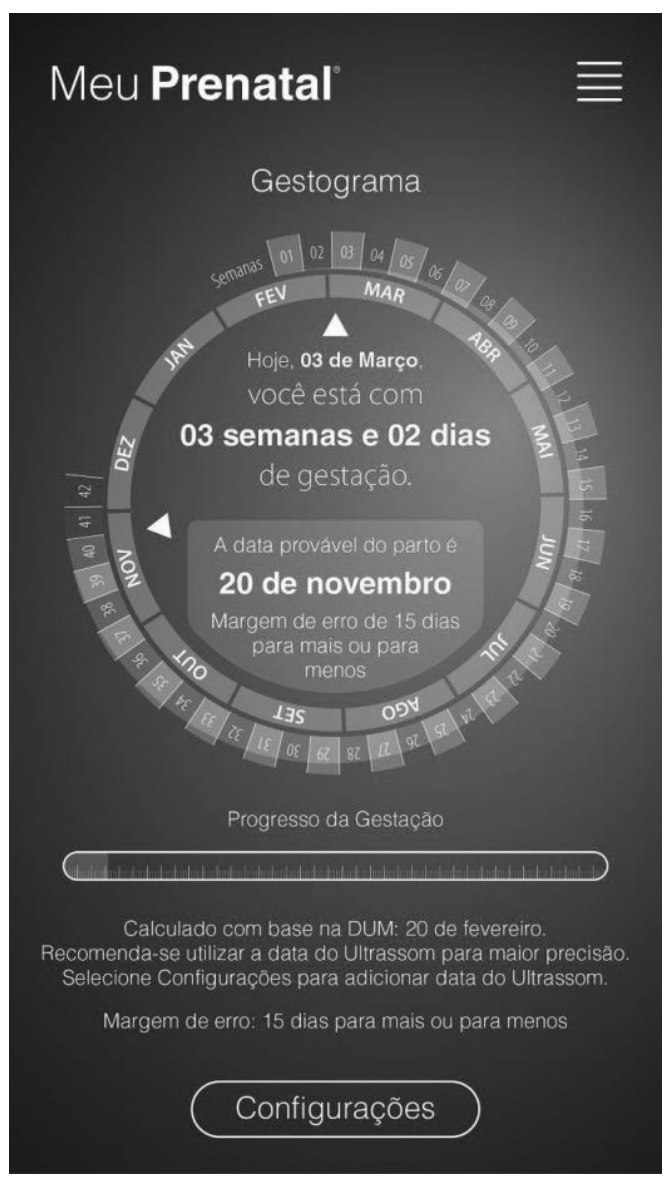

Figura 1. Interface principal do APP Meu Pré-natal apresentando um gestograma com detalhamento sobre a cronologia da gravidez.

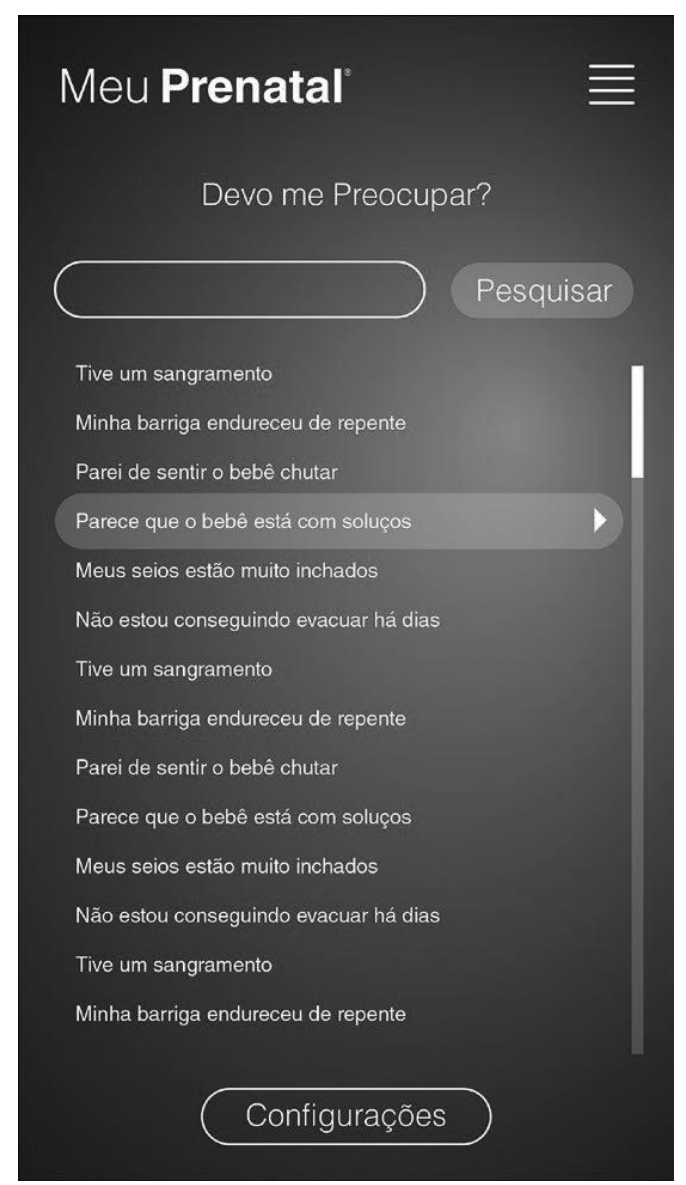

Figura 2. Interface do tipo pergunta-resposta, acessível pela opção Seção de Dúvidas - Devo me

Preocupar, do menu do APP Meu Pré-natal.

Como previsto no plano de desenvolvimento do projeto, as orientações educativas permearam todo APP. No formato de pergunta objetiva e resposta curta (Figura 2), cada item de interesse encontra-se acessível a partir do menu Seção de Dúvidas. Essa seção foi organizada em cinco subseções, seguindo ordem cronológica da gravidez: Minha gravidez (72 itens); Devo me preocupar? (32 itens); Chegou a hora? (18 itens); O bebê nasceu (26 itens); Fique por dentro (97 itens), Tabela 1. Além desta seção estar disponível para consulta no dispositivo móvel também em modo offline, o APP envia em sequência cronológica uma mensagem semanal para o usuário: 25 itens no primeiro trimestre, 26 itens no segundo trimestre, 17 itens no terceiro trimestre, 16 itens entre 37 semanas de gravidez e o parto e 12 itens no pós-parto.

Tabela 2. Tema central do conjunto pergunta-resposta dos itens de conteúdo educativo preparados e validados por especialistas para o APP Meu Pré-natal.

\begin{tabular}{|c|c|}
\hline Temas & Número de itens \\
\hline Alimentação e nutrição & 7 \\
\hline Amamentação & 12 \\
\hline Câncer do colo do útero & 1 \\
\hline Certeza de gravidez & 1 \\
\hline Contracepção e puerpério & 5 \\
\hline
\end{tabular}




\begin{tabular}{|c|c|}
\hline Cronologia da gravidez & 6 \\
\hline Direitos na gravidez & 6 \\
\hline Fisiologia materno-fetal & 18 \\
\hline Hábitos de vida, mitos, vícios & 11 \\
\hline Higiene e beleza & 7 \\
\hline O cuidado pré-natal & 3 \\
\hline Parto e nascimento & 4 \\
\hline Parto prematuro & 1 \\
\hline Plano de parto & 1 \\
\hline Sinais de perigo & 9 \\
\hline Triagem neonatal & 3 \\
\hline Zika vírus & 2 \\
\hline TOTAL & $\mathbf{9 7}$ \\
\hline
\end{tabular}

No formato de vídeo com cinco minutos de duração, informações relevantes sobre preparação para o parto e pósparto imediato foram implementados na aplicação. Os temas abordados no formato animação com legenda e narração incluiu: os diretos da gestante, documentos e itens na mala a serem levados para maternidade, orientações para internação para o parto e sinais de alerta, modalidades de assistência obstétrica e cuidados imediatos no puerpério. $O$ documento é parte integrante do APP e pode ser também assistido no modo em modo off-line, ou seja, mesmo que o dispositivo esteja sem conexão com a Internet.

De forma complementar às informações, um conjunto de funcionalidades foi implementado. Um contador de contrações programado monitorar dez minutos de observação foi oferecido para orientar a melhor hora de procurar a maternidade para o parto (Brasil, 2014a). Nas seções Álbum de Fotos e Dados do Bebê as usuárias puderam capturar imagens do ambiente a partir da câmera do dispositivo móvel. Adicionalmente, para que toda a experiência da gestante pudesse ser documentada, o APP produz um relatório contendo dados sobre o nascimento e fotos capturadas periodicamente pelas usuárias, mostrando todo o progresso da gestação. Esse relatório pode ser compartilhado através da Internet, seja pelo e-mail ou através da mídias sociais.

Com a publicação em outubro de 2016 e divulgação nas mídias locais, a aplicação atingiu em julho de 2017: 19.364 downloads. A maior parte a partir de dispositivos de comunicação utilizados em sua captura nas lojas virtuais foi do tipo Android: $14.791(76,4 \%)$ e $4.573(23,3 \%)$ no sistema operacional iOS. Os usuários foram provenientes de 81 países, entre eles o Brasil com 18.030 (93,1\%), Estados Unidos da América com 249 (1,3\%), Portugal com 233 (1,2\%) e 115 (0,59\%) Angola, 90 (0,46\%) México, 88 (0,45\%) Japão, 39 (0,20\%) Moçambique, 30 (0,15\%) Suíça, entre outros.

A propriedade intelectual do programa de computador foi depositada no Instituto Nacional de Propriedade Intelectual (INPI) brasileiro em outubro de 2016, sob identificação BR 5120160013141.

\section{Discussão}

A informática médica é área do conhecimento essencialmente interdisciplinar e seu potencial transformador de práticas cotidianas é um tema que tem atraído a atenção de cientistas, políticos, gestores e da própria sociedade (Shekelle, Morton, \& Keeler, 2006). No que se refere a saúde, a comunicação já é considerada um dos pontos mais relevantes da prestação de cuidados, assim constitui-se como numa importante ferramenta de gestão dos serviços e das próprias políticas públicas do setor (Organization, 2006). O Brasil acaba de consolidar o dispositivo móvel como principal meio de acesso à Internet, ultrapassando também a cifra de um aparelho por habitante (Brasil, 2016). A investigação sobre o uso das TICs realizada pela Pesquisa Nacional por Amostra de Domicílios deixou clara a capacidade de comunicação através dos dispositivos móveis, em todas as classes sociais e idades da população brasileira. Em todas as cinco grandes regiões brasileiras, avaliadas por amostragem em 2015, o acesso à Internet via banda larga atingiu 99\% dos domicílios conectados (Brasil, 2016). A presente proposta apresentou uma experiência de sucesso na disseminação de informações educativas sobre a gravidez, utilizando-se do cenário tecnológico e da conectividade caraterísticos da sociedade atual. O diferencial do projeto aqui apresentado é a qualidade das 
informações disponibilizadas, o caráter acadêmico e a gratuidade do serviço para a população. Para isso, durante o desenvolvimento e validação do software o grupo multidisciplinar envolvido preocupou-se com os domínios de qualidade para o APP: base clínica e científica, usabilidade, impacto e popularidade (Grundy, Wang, \& Bero, 2016).

Em contraponto, com o crescimento exponencial das TICs, enfrenta-se uma exposição exagerada às informações, sejam elas benéficas ou não, muitas vezes exageradas e prejudiciais (Martins \& Duarte, 2016). É preciso mais do que uma visão crítica sobre a cifra de quarenta mil APP sobre bem-estar e saúde disponíveis nas lojas de aplicativos em todo mundo (Carecloud, 2016). É preciso que se discuta a sua regulamentação. Tal disponibilização não se acompanhou de um monitoramento de qualidade, o que tem sido preocupação dos governos, estudos científicos e revisões sistemáticas (Grundy, Wang, \& Bero, 2016; Zhao, Freeman, \& Li, 2016). Além disto, é legitimo o direito à privacidade, preservando-se a liberdade de fornecer informações em APP e ambiente virtual. Para isso, o Termo de Adesão deixa claro os propósitos do projeto, apresenta informações sobre a origem das orientações e a importância de se priorizar o cuidado prestado diretamente pelo profissional de saúde qualificado para o pré-natal. 0 direito de não informar seus dados foi respeitado, uma vez que não são obrigatórios.

O APP foi desenvolvido utilizando-se um modelo conhecido como híbrido. Esse método foi escolhido devido à maior velocidade trazida para se satisfazer o requisito de compatibilidade com sistemas operacionais Android e iOS, de maneira rápida e eficiente. Como o próprio conceito diz, o desenvolvimento híbrido traz a possibilidade de programação de aplicações multiplataforma nos quais apenas um tipo de código o APP é desenvolvido e publicado nas principais lojas de aplicativos móveis. Esse ganho em relação ao tempo de desenvolvimento se deve ao fato dispensar conhecimentos para mais de uma arquitetura e linguagem de programação, no caso, Java e Objectivec/Swift respectivamente, o que implicaria praticamente no desenvolvimento de dois sistemas completamente independentes.

Quanto à experiência formativa para os envolvidos, em todo ciclo de vida do software, a participação interdisciplinar para construção coletiva do sistema e o monitoramento de sua disseminação na sociedade trouxe ganhos inegáveis. Os temas abordados proporcionaram uma discussão construtiva sobre o modelo de assistência obstétrica em um país com elevadas taxas de parto cesariana e desafios ainda por vencer, no que se refere à qualidade do cuidado materno-infantil (Reis et al., 2011, Brasil, 2014b). O desenho do produto teve foco na disseminação do saber produzido no âmbito da universidade, em linguagem acessível às gestantes. Todos os esforços foram empenhados para que a facilidade de comunicação por meio eletrônico fosse preservada, empregando-se textos claros e curtos, mas de conteúdo sólido e objetivo. No que se refere aos direitos das gestantes e familiares, as regras atuais no país foram consultadas e permearam as orientações.

O impacto na sociedade foi rápido a partir do lançamento, mesmo aferido logo nos seis primeiros meses. A rápida popularização e disseminação do APP em vários países é evidência da importância desse canal de comunicação para propostas de educação em saúde. A identificação de pontos do cuidado que merecem intervenção é considerada uma das estratégias para que um APP possa produzir mudanças benéficas no comportamento de seus usuários (Zhao, Freeman, \& Li, 2016). Foi esse o propósito já na fase de projeto que buscou no contato direto com as gestantes perceber suas necessidades e dúvidas. Tal framework baseado na análise de um cenário real do cuidado antenatal proporcionou a toda equipe do projeto a possibilidade de se ajustar o conteúdo de interesse, as funcionalidades de acordo com as demandas da gestante, além da possibilidade de testar previamente a linguagem para os textos do APP. No entanto, não foi possível inferir o quanto as intervenções educativas, as funcionalidades preparadas para orientar a melhor de procurar a maternidade ou alertas para anormalidades impactam o cuidado pré-natal ou mesmo se álbum de recordações com fotografias serviu para tornar a experiência da gravidez única para cada casal. Mas no futuro ainda é possível considerar esse tipo de validação. Ainda assim, acreditamos em seu potencial de promover interação transformadora da informação junto às gestantes, envolvendo uma estratégia de TIC para promoção da saúde. A possibilidade de atuar antes do nascimento para identificar riscos e amenizar fatores que trazem complicações para a saúde da mulher e do bebê é motivação suficiente para envolver profissionais de saúde e da informática neste tipo de desafio tecnológico. 


\section{Agradecimentos}

O estudo foi realizado com suporte da Fundação de Amparo à Pesquisa de Minas Gerais (FAPEMIG) e da Fundação Bill \& Melinda Gates, através o Projeto Newborn skinage. Os autores agradecem as contribuições dos estudantes de medicina: Laura Drummond Nogueira, Laura Campos, Jessica Ferreira, lara Fantini, Igor Braga, Henrique Lima e das Professoras Kleyde Ventura e Patrícia Gomes Teixeira e ao designer Dimitri B. Santos.

\section{Referências}

ACOG. (2014). Committee opinion no 611: method for estimating due date. Obstet Gynecol, 124(4), 863-866. doi:10.1097/01.AOG.0000454932.15177.be.

Brasil. (2014a). Caderneta da Gestante. edited by Ministério da Saúde. Brasília. Distrito Federal: Brasil, Ministério da Saúde. Caderneta da Gestante 2014. Brasília. Distrito Federal.

Brasil. (2014b). Objetivos de Desenvolvimento do Milênio: Relatório Nacional de Acompanhamento. In, ed Instituto de Pesquisa Econômica Aplicada e Secretaria de Planejamento e Investimentos Estratégicos. Brasília: Grupo Técnico para o acompanhamento dos ODM (accessed 14/04/2017).

Brasil. (2016). Acesso à internet e à televisão e posse de telefone móvel celular para uso pessoal: 2015. In, ed IBGE Instituto Brasileiro de Geografia e Estatística. Rio de Janeiro: IBGE, Coordenação de Trabalho e Rendimento. Retrieved from http://biblioteca.ibge.gov.br/visualizacao/livros/liv99054.pdf (accessed 04/23/2017).

Brasil, Ministério da Saúde, \& Departamento de Ações Programáticas Estratégicas. (2007). Política Nacional de Atenção Integral à Saúde da Mulher Princípios e Diretrizes. edited by Ministério da Saúde. Brasília: Ministério da Saúde.

Brasil, P., Pereira Jr, J., Moreira, M., Nogueira, R., Damasceno, L., Wakimoto, M., Rabello, R., Valderramos, S., Halai, U., Salles, T. (2016). Zika virus infection in pregnant women in Rio de Janeiro. New England Journal of Medicine, 375(24), 2321-2334\%@ 0028-4793.

Butt, K., Lim, K., Bly, S., Cargill, Y., Davies, G., Denis, N., Hazlitt, G., Morin, L., Ouellet, A., Salem, S. (2014). Determination of gestational age by ultrasound. Journal of Obstetrics and Gynaecology Canada, 36(2), 171-181.

Carecloud. (2016). Stratategic insights, perspectives and industry trends for healthcare executives. Continuum, accessed 24/04/2017. http://www.carecloud.com/continuum/7-best-fda-approved-health-apps/.

Chen, E., \& Mangone, E. (2016). A Systematic Review of Apps using Mobile Criteria for Adolescent Pregnancy Prevention (mCAPP). JMIR mHealth and uHealth, 4(4).

Fonseca, A. R., \& Alencar, M. S. M. (2016). O uso de aplicativos de saúde para dispositivos móveis como fontes de informação e educação em saúde. Anais do SNBU \%@ 2359-6058.

Grundy, Q. H., Wang, Z., \& Bero, L. A. (2016). Challenges in Assessing Mobile Health App Quality: A Systematic Review of Prevalent and Innovative Methods. American Journal of Preventive Medicine, 51(6), 1051-1059 \%@ 07493797.

Grünebaum, A., McCullough, L. B., Sapra, K. J., Brent, R. L., Levene, M. I., Arabin, B., \& Chervenak, F. A. (2014). Early and Total Neonatal Mortality in Relation to Birth Setting in the United States, 2006-2009. American journal of obstetrics and gynecology.

Islam, S. M. R., Kwak, D., Kabir, M. D. H., Hossain, M., \& Kwak, K. (2015). The internet of things for health care: a comprehensive survey. IEEE Access 3:678-708 \%@ 2169-3536.

Kwak, H., Lee, C., Park, H., \& Moon, S. (2010). What is Twitter, a social network or a news media?

Laurenti, R., Siqueira, A. A. F., Jorge, M. H. P. M., Gotlieb, S. L. D., \& Pimentel, E. C. (2013). Perinatal mortality in hospitals of the state of São Paulo: methodological aspects and some characteristics of mothers and conceptuses. Journal of Human Growth and Development, 23(3), 261-269. 
Martins, F. B., \& Duarte, H. G. (2016). O direito ao esquecimento: a influência da informação na vida social. Revista Vox, 1(2),11-27\%@ 2359-5183.

Moraes, C. L., \& Reichenheim, M. E. (2000). Validade do exame clínico do recém-nascido para a estimação da idade gestacional: uma comparação do escore New Ballard com a data da última menstruação e ultra-sonografia. Cad Saúde Pública, 16(1), 83-94.

Organization, World Health. (2006). Building foundations for eHealth: progress of member states: report of the Global Observatory for eHealth: OPS.

Patah, L. E. M., \& Malik, A. M. (2011). Modelos de assistência ao parto e taxa de cesárea em diferentes países. Rev Saúde Pública, 45(1), 185-94.

Reis, Z. S. N., Pereira, A. C., Correia, R. J. C., Freitas, J. A. S., Cabral, A. C. V., \& Bernardes, J. (2011). Análise de indicadores da saúde materno-infantil: paralelos entre Portugal e Brasil. Revista Brasileira de Ginecologia e Obstetrícia, 33, 234-239.

Shekelle, P., Morton, S. C., \& Keeler, E. B. (2006). Costs and benefits of health information technology.

Souza, E. C. B. (2017). Os direitos e deveres de gestantes atendidas no Sistema Único de Saúde.

Tibes, C. M. S., Dias, J. D., \& Zem-Mascarenhas, S. H. (2014). Aplicativos móveis desenvolvidos para a área da saúde no Brasil: revisão integrativa da literatura. Revista Mineira de Enfermagem 18 (2), 471-486 \%@ 1415-2762.

UNICEF. (2011a). Guia dos Direitos da Gestante e do Bebê. In [ilustrações de Ziraldo], ed Globo. São Paulo: Fundo das Nações Unidas para a Infância https://www.unicef.org/brazil/pt/br_guiagestantebebe.pdf.

UNICEF. (2011b). Guía para la atención de la salud integral de la niñez - adolescencia y madre embarazada. In Vigilancia del crescimiento y desarrollo, ed Organización Panamericana de La Saude. Asunción: Ministerio de Salud Pública y Bienestar Social. Dirección General de Programas de Salud. http://www.paho.org/can/index.php?option=com_docman\&view=download\&category_slug=paraguay1\&alias=6534-guia-para-la-atencion-de-la-salud-integral-de-la-ninez-adolescencia-y-de-la-madreembarazada\&ltemid $=308 \&$ lang $=$ es.

Vettore, M., \& Lamarca, G. (2012). Atenção pré-natal no Brasil: uma questão de oferta, de acesso ou de escolaridade materna. Determinantes Sociais da Saúde. Portal e Observatório sobre Iniquidades em Saúde [Internet].

Young, M. F., Nguyen, P. H., Addo, O. Y., Pham, H., Nguyen, S., Martorell, R., \& Ramakrishnan, U. (2017). Timing of Gestational Weight Gain on Fetal Growth and Infant Size at Birth in Vietnam. PloS one, 12(1), e0170192.

Zhao, J., Freeman, B., \& Li, Mu. (2016). Can mobile phone apps influence people's health behavior change? An evidence review. Journal of Medical Internet Research, 18(11), e287.

\section{Sobre os Autores}

Zilma Silveira Nogueira Reis (PhD) é médica ginecologista e obstetra (1990), Mestre em Medicina (1993) e Doutora em Medicina (2002) pela Universidade Federal de Minas Gerais, quando obteve bolsa de doutorado-sanduiche na Alemanha, PROBRAL 113/00 (CAPES/DAAD). Realizou Estágio Sênior Pós-Doutoral no Exterior com bolsa CAPES, na Universidade do Porto, Portugal (2011). Atualmente é Professora Associada do Departamento de Ginecologia e Obstetrícia, do Programa de Pós-graduação em Saúde da Mulher da UFMG e Médica do Hospital das Clínicas. Coordena o Centro de Informática Médica da Faculdade de Medicina da UFMG desde 2012. É Vice-presidente da Sociedade Brasileira de Informática em Saúde (SBIS).

Júlia Relva Basso possui graduação em História pela Universidade de São Paulo (2008). Brasileira. Aluna em graduação do Curso de Medicina da Universidade Federal de Minas Gerais, Brasil. Atuou no Projeto de Extensão Meu Pré-natal Siex 401714, desenvolvendo a aplicação para dispositivos móveis.

Marcelo R. Santos Junior é cientista da Computação pela Pontifícia Universidade Católica de Minas Gerais (2017), Brasil. Atua nas áreas de Inteligência Artifical, Mineração de Dados, Informática em Saúde e Desenvolvimento para dispositivos móveis. Atuou no Projeto de Extensão Meu Pré-natal Siex 401714, desenvolvendo a aplicação para dispositivos móveis, com bolsa Fapemig AUC-00032-15. Mestrando do Programa de Pósgraduação em Saúde da Mulher, Universidade Federal de Minas Gerais, Brasil. 
Isaias José Ramos de Oliveira (MsC) possui graduação em Ciência da Computação pela Universidade Federal de Minas Gerais (2010) e mestrado em Medicina (Obstetrícia e Ginecologia) pela Universidade Federal de Minas Gerais (2016). Atualmente é sócio - Butec Inovação llimitada e técnico em tecnologia da informação da Universidade Federal de Minas Gerais. Tem experiência na área de Ciência da Computação, com ênfase em Ciência da Computação, atuando principalmente nos seguintes temas: atividades educacionais, educação em saúde, métodos contraceptivos, planejamento familiar e aplicativos móveis.

Wagner Bento Magalhães possui graduação em redes de computadores pela Universidade Estácio de Sá, Brasil. Atua como analista em Redes de Computadores do Centro de Informática em Saúde da Faculdade de Medicina, Universidade Federal de Minas Gerais, Brasil. Pesquisador na área de informática médica do Núcleo de Informática em Saúde desta Faculdade. Atualmente trabalhando na área de instrumentação não invasiva em engenharia biomédica, dentro do projeto Light Scan Skin Age. Mestrando do Programa de Pós-graduação em Saúde da Mulher, Universidade Federal de Minas Gerais, Brasil.

Juliano de Souza Gaspar (PhD) é doutorado em Informática Médica pelo programa de Pós-Graduação da Saúde da Mulher da Faculdade de Medicina da UFMG. Concluiu o Mestrado em Informática Médica pela Faculdade de Medicina da Universidade do Porto (2011), após obter o Bacharelato em Ciências da Computação pela Universidade do Vale do Itajaí (2006). É membro do núcleo de Informática em Saúde da Faculdade de Medicina da UFMG, onde desenvolve atividade de investigação científica na área da saúde e a informática, participa como educador nos grupos de iniciação científica e pesquisa, bem como na elaboração e publicação de artigos. Leciona as disciplinas de Bioestatística, Epidemiologia, Sistemas de Informação em Saúde e Informática Médica. Desde 2009 é membro do CINTESIS (Centro de Investigação em Tecnologias e Sistemas de Informação em Saúde da Faculdade de Medicina da Universidade do Porto, Portugal), onde realiza atividades de pesquisador científico. Trabalhou 13 anos em empresas privadas na área de informática, suas principais atividades foram como Analista de Sistemas e Coordenação de Projetos, tendo também atuado como instrutor de informática e designer gráfico.

Alamanda Kfoury Pereira é médica ginecologista e obstetra. Professora Titular do Departamento de Ginecologia e Obstetrícia da Faculdade de Medicina da Universidade Federal de Minas Gerais. Graduação em Medicina pela UFMG (1986), Mestrado em 1990 e Doutorado em 1998, em Obstetrícia e Medicina Fetal, no Programa de Pós Graduação em Saúde da Mulher, Faculdade de Medicina da Universidade Federal de Minas Gerais. Na coordenação do colegiado do curso de Medicina, coordenou o processo de implantação do novo currículo médico em versão 2014 e a criação em 2014 do Núcleo Docente Estruturante. Vice-diretora da Faculdade de Medicina da Universidade Federal de Minas Gerais. 